Pacific Journal of Mathematics

TOPOLOGICAL MEASURE THEORY FOR COMPLETELY 


\title{
TOPOLOGICAL MEASURE THEORY FOR COMPLETELY REGULAR SPACES AND THEIR PROJECTIVE COVERS
}

\author{
ROBERT F. WHEELER
}

This paper investigates the relationships among tight, $\tau$-additive, and $\sigma$-additive Baire measures on a completely regular Hausdorff space $X$ and its projective cover $E(X)$. The most interesting questions arise in the $\sigma$-additive case, and lead to the following definitions: the space $X$ has the weak (resp. strong) lifting property if for each $\sigma$-additive measure on $X$, some (resp., every) pre-image measure on $\mathbf{E}(X)$ is $\sigma$-additive. It is shown that every weak $c b$ space has the strong lifting property, while the Dieudonné plank fails even the weak lifting property. Also, if $X$ is weak $c b$, then $X$ is measure-compact if and only if $E(X)$ is measurecompact.

Some applications to extensions of measures on lattices and to strict topologies on spaces of continuous functions are given. A relationship between the lifting properties mentioned above and conventional use of the term "lifting" in measure theory is indicated.

A topological space is said to be extremally disconnected if the closure of every open set is again open. Such a property seems remote from the topological settings usually encountered in analysis; for example, a metric space with this property must be discrete. Nonetheless, the property of extremal disconnectedness occurs with surprising frequency in many basic results of modern analysis. Here are some of them:

(1) The lattice $C(X)$ of continuous real-valued functions on a completely regular space $X$ is Dedekind complete if and only if $X$ is extremally disconnected.

(2) A Boolean algebra is complete if and only if its Stone space is extremally disconnected.

(3) If $X$ is a compact Hausdorff space, then $C(X)$ with the supremum norm is isometrically isomorphic to a dual Banach space if and only if $X$ is hyperstonian (i.e., extremally disconnected, and the union of the supports of the normal measures on $X$ is dense in $X)$.

This paper is concerned with Baire measures on completely regular spaces. The critical fact which motivates the work is that for each completely regular Hausdorff space $X$, there is an extremally disconnected space $E(X)$, called the projective cover or absolute of $X$, and a perfect irreducible map $\kappa$ of $E(X)$ onto $X$. We can 
observe at once that (1) $f \rightarrow f \circ \kappa$ is an isometric embedding of $C^{*}(X)$ in $C^{*}(E(X))$; and (2) the adjoint $\kappa^{*}$ of this embedding maps $M(E(X))$, the space of finitely-additive Baire measures on $E(X$,$) onto$ $M(X)$.

A principal focus of topological measure theory is the delineation and study of certain subspaces of $M(X)$ - notably the tight measures $\left(M_{t}\right), \tau$-additive measures $\left(M_{\tau}\right)$ and $\sigma$-additive measures $\left(M_{\sigma}\right)$. For example, a space $X$ is said to be measure-compact if $M_{\sigma}(X)=M_{\tau}(X)$, and one seeks purely topological conditions for this to occur; it is known that the Lindelöf property is sufficient, and realcompactness is necessary.

The plan of attack in this paper is to relate corresponding spaces of measures on $X$ and its projective cover: for example, $M_{\tau}(X)$ and $M_{\tau}(E(X))$. There is a basic reason for doing this: in extremally disconnected spaces, the Baire sets have a relatively simple structure. For example, the zero-sets of continuous realvalued functions are precisely the countable intersections of clopen sets. This suggests that a topological formulation of measurecompactness (and other concepts of similar type) may be easier to obtain for extremally disconnected spaces than in the general setting. Then, via the correspondences mentioned above, the results could be extended to all completely regular spaces.

We summarize the principal results of the paper as follows: for $M_{t}$ and $M_{\tau}$ there is an exact correspondence between $X$ and $E(X)$, in the sense that $\kappa^{*-1}\left(M_{t}^{+}(X)\right)=M_{t}^{+}(E(X))$, and $\kappa^{*-1}\left(M_{\tau}^{+}(X)\right)=$ $M_{\tau}^{+}(E(X))$. The situation is much more interesting and complicated for $M_{\sigma}$, and we are led to the following definitions:

Definition 1. $X$ has the weak lifting property (WLP) if for each $\mu \in M_{\sigma}^{+}(X), \exists \nu \in M_{\sigma}^{+}(E(X))$ with $\kappa^{*} \nu=\mu$.

DEFINITION 2. $X$ has the strong lifting property (SLP) if for each $\mu \in M_{o}^{+}(X)$, every pre-image of $\mu$ in $M^{+}(E(X))$ is in $M_{o}^{+}(E(X))$ (i.e., $\kappa^{*-1}\left(M_{\sigma}^{+}(X)\right)=M_{\sigma}^{+}(E(X))$ ).

We find examples of (1) a space which fails the WLP and (2) a space which has the WLP but fails the SLP. These examples emphasize the almost realcompact spaces introduced by Frolik [10] and recently studied by Kato [18]. The notion of a weak $c b$ space, introduced by Mack and Johnson [25] in their study of the lattice completion of $C(X)$, is characterized in a new way, leading to the principal positive result of the paper: If $X$ is weak $c b$, then $X$ has the SLP. As a corollary, if $X$ is weak $c b$, then $X$ is measurecompact if and only if $E(X)$ is measure-compact.

The major question raised and left unresolved by this paper 
seems to be: if $X$ is realcompact, must $X$ have the weak (or strong) lifting property? In investigating this problem we find a relationship between the lifting properties defined above and the conventional use of the term "lifting" in measure theory, via the density topology on the real line.

As applications of our results, we analyze a recent paper by Sultan [39] on extensions of measures in our setting, and also examine the relationships among the various strict topologies on $C^{*}(X)$ and $C^{*}(E(X))$.

In closing this introductory section, several general remarks seem to be in order:

(1) The relationship between normal measures on $X$ and $E(X)$ has been studied by Lacey and Hebert [22], Flachsmeyer [9], and others, and a very complete and satisfying theory has been obtained.

(2) Rosenthal [32] has obtained strong results on measures on extremally disconnected compact spaces; his work has been extended and simplified by Kupka [21] and others. It was in the course of studying these results that the author came to feel that $E(X)$ should play a significant role in the study of measures on $X$.

(3) Recent developments in topological measure theory have tended to stress the embedding of $X$ as a subspace of its StoneCech compactification $\beta X$. In this paper the emphasis is reversed, since $X$ is the range, rather than the domain, of the map $\kappa$. Nonetheless, the equality $E(\beta X)=\beta E(X)$ allows us to make good use of the basic $X$ vs. $\beta X$ theory.

Finally, the author thanks Grant Woods for a number of very helpful conversations about projective covers.

1. Notation and preliminary remarks. A basic reference for topological measure theory is Varadarajan [43]. We shall also refer to more recent work of Knowles [19], Moran [28, 29], Mosiman and Wheeler [30], and Sentilles [35]. Throughout the discussion $X$ denotes a completely regular Hausdorff space, and $C^{*}(X)$ is the space of bounded continuous real-valued functions on $X$. If $\mu \in M(X)$, then $\mu$ is (a) $\sigma$-additive, if $\mu\left(Z_{n}\right) \rightarrow 0$ for every decreasing sequence $\left(Z_{n}\right)$ of zero-sets with empty intersection; (b) $\tau$-additive, if the corresponding result holds for nets of zero-sets which decrease (compatibly with the partial order) and have empty intersection; (c) tight, if for every $\varepsilon>0$ there is a compact subset $K$ of $X$ with $\mu_{*}(X-K)<\varepsilon$. As usual, $M_{\sigma}(X), M_{\tau}(X)$, and $M_{t}(X)$ denote the collections of $\sigma$ additive, $\tau$-additive, and tight measures on $X$. It is well-known that $M_{t} \subset M_{\tau} \subset M_{\sigma}$.

Each $\mu \in M(X)$ gives rise (via the identification of $C^{*}(X)$ and $\left.C^{*}(\beta X)\right)$ to a corresponding regular Borel measure $\nu$ on $\beta X$. A non- 
negative measure $\mu$ is $\sigma$-additive (resp. $\tau$-additive) if and only if the corresponding $\nu$ vanishes on all zero-sets (resp. compact sets) of $\beta X-X$ [19].

A map $f: X \rightarrow Y$ is said to be perfect if $f$ is continuous, closed, and onto, and $f^{-1}(y)$ is compact for all $y \in Y$. Also, $f$ is said to be irreducible if the image of every proper closed subset of $X$ is a proper subset of $Y$.

The projective cover (or absolute) of $X$ is an extremally disconnected space $E(X)$ and a perfect irreducible map $\kappa$ of $E(X)$ onto $X$. The construction of the absolute has been extended to successively larger classes of spaces by Gleason [12], Strauss [38], and the Russian school [31]. Some of the deepest results on extremally disconnected spaces can be found in Efimov [8]. The author has found the most valuable single reference in obtaining a working knowledge of projective covers to be a sequence of papers by Grant Woods and co-authors [46-50]. Relying on these sources for details, we collect here the basic results about $E(X)$ that we need.

A subset $F$ of $X$ is regular closed if $F=\operatorname{cl}_{X}$ int $_{X} F$. The family $R C(X)$ of regular closed subsets of $X$ is a complete Boolean algebra [36] under the operations: $\bigvee_{\alpha} F_{\alpha}=\operatorname{cl}_{X}\left(\cup F_{\alpha}\right), \Lambda_{\alpha} F_{\alpha}=\operatorname{cl}_{X} \operatorname{int}_{X}\left(\bigcap_{\alpha} F_{\alpha}\right)$, $F^{\prime}=\operatorname{cl}_{X}(X-F)$. The map $F \rightarrow \operatorname{cl}_{\beta X} F$ is a Boolean isomorphism of $R C(X)$ onto $R C(\beta X)$. Let $S$ be the Stone space of $R C(\beta X)$, interpreted as the set of ultrafilters of regular closed subsets of $\beta X$. Note that "filter" here is in the Boolean sense: $F, G \in \mathscr{F} \Rightarrow F \wedge G \in \mathscr{F}$. Let $\bar{\lambda}: R C(\beta X) \rightarrow \operatorname{clop}(S)$ be the canonical correspondence between regular closed subsets of $\beta X$ and clopen subsets of $S$. There is a natural map $\bar{\kappa}: S \rightarrow \beta X$ which sends each ultrafilter of regular closed sets to its limit in $\beta X$. Then $S$ is extremally disconnected and $\bar{\kappa}$ is perfect and irreducible, so $S=E(\beta X)$.

Now let $T=\bar{\kappa}^{-1}(X) \subset S$. Then $T$ is dense in $S$, so $T$ is extremally disconnected, and $\beta T=S[11,6 M]$. If $\kappa=\bar{\kappa} \mid T$, then $\kappa$ is perfect and irreducible, so $T=E(X)$. It follows that $\beta E(X)=E(\beta X)$.

We have then the commutative diagram:

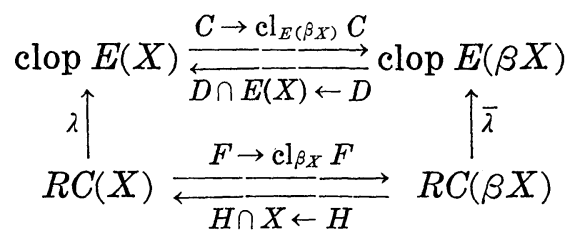

where each connecting map is a Boolean isomorphism. Intuitively, $E(X)$ is the set of all ultrafilters of regular closed subsets of $X$ which converge to a point of $X ; \kappa$ sends each such ultrafilter to its limit. If $F_{0} \in R C(X)$, then $\lambda\left(F_{0}\right)=\left\{p \in E(X): F_{0} \in p\right\}$ is clopen in $E(X)$. 
Then $\kappa\left(\lambda\left(F_{0}\right)\right)=F_{0}$, but $\lambda\left(F_{0}\right) \varsubsetneqq \kappa^{-1}\left(F_{0}\right)$ in general. The reason for this is as follows: if $x \in X, p \in E(X)$, and $\kappa(p)=x$, then $p$ must contain all regular closed sets $F$ for which $x \in$ int $_{X} F$. However, if $x \in F_{0}-\operatorname{int}_{X} F_{0}$, then $x \in F_{0}^{\prime}$. Choosing an ultrafilter $p_{0}$ which refines $\left\{F: x \in \operatorname{int}_{x} F\right\} \cup\left\{F_{0}^{\prime}\right\}$, we have $\kappa\left(p_{0}\right)=x$, so $p_{0} \in \kappa^{-1}\left(F_{0}\right)$, but $p_{0} \notin \lambda\left(F_{0}\right)$.

The exact relationship here is: $\operatorname{cl}_{E(X)}\left(\kappa^{-1}\left(\operatorname{int}_{X} F\right)\right)=\operatorname{int}_{E(X)} \kappa^{-1}(F)=$ $\lambda(F) \subset \kappa^{-1}(F)$ [22]. The inclusion is an equality if and only if $F$ is clopen. The reader may find it instructive to consider the following example: $X=\hat{N}$, the one point compactification of $N, E(X)=\beta N$, $\kappa: E(X) \rightarrow X$ sends each integer to itself and $\beta N-N$ to $\infty, F=$ $\{$ evens $\} \cup\{\infty\} \in R C(X)$. Then $\kappa^{-1}\left(\right.$ int $\left._{X} F\right)=\{$ evens $\} \varsubsetneqq \lambda(F)=\operatorname{cl}_{\beta N}\{$ evens $\} \varsubsetneqq$ $\kappa^{-1}(F)=\{$ evens $\} \cup\{\beta N-N\}$.

The following result (essentially Lemma 2.4 of [48]) will be very useful: if $\left(C_{n}\right)$ is a decreasing sequence of clopen sets in $E(X)$, then

$$
\kappa\left(\bigcap_{1}^{\infty} C_{n}\right)=\bigcap_{1}^{\infty} \kappa\left(C_{n}\right)
$$

$\kappa$ is not an open map, but it is closed and irreducible, so if $U$ is a nonempty open subset of $E(X)$, then $\left\{x \in X: \kappa^{-1}(x) \subset U\right\}=X-$ $\kappa(E(X)-U)$ is a nonempty open subset of $X$.

2. $X$ vs. $E(X)$. There are many properties which $X$ and $E(X)$ always have in common. We list some of them.

Theorem 1. For the following topological properties $P, X$ has $P$ if and only if $E(X)$ has $P$ : (a) compact (b) $\sigma$-compact (c) Lindelöf (d) countably compact (e) locally compact (f) paracompact (g) countably paracompact (h) metacompact (i) pseudocompact (j) k-space (k) separable (l) countable chain condition (m) dense in itself.

Proof. (a)-(g): [13]; (h): $X$ metacompact $\Rightarrow E(X)$ metacompact is an easy exercise [7, p. 254]; proofs of the converse can be found in [17] and [51]; (i): [48]; (j): [2]; (k): follows from the irreducibility of $\kappa$; (l): follows from the last sentence of the previous section; $(\mathrm{m})$ : if $x_{0}$ is an isolated point of $X$, then there is a unique ultrafilter $p$ of regular closed sets converging to $x_{0}$, and $\kappa^{-1}\left(x_{0}\right)=\{p\}$ is clopen in $E(X)$. Conversely, if $p$ is an isolated point of $E(X)$, with $\kappa(p)=x$, then $\{x\}$ is a regular closed set, so $x$ is an isolated point of $X$.

Let us remark that (a)-(h) and (j) are valid for any perfect map $f: X \rightarrow Y$.

There is one more property which belongs in Theorem 1, and which plays an important role in the sequel. A space is almost realcompact [10] if every ultrafilter of regular closed sets such that 
countable subfamilies have nonempty intersection is fixed. It is known that realcompact implies almost realcompact, but not conversely [18]. Almost realcompactness is preserved by closed subsets and by products, from [14], every $X$ admits an "almostrealcompactification" $a X$, with $X \subset a X \subset \nu X \subset \beta X$. This construction has been examined in detail by Woods [50]. If $f: X \rightarrow Y$ is perfect, then $X$ is almost realcompact if and only if $Y$ is almost realcompact [10]. But for extremally disconnected spaces, it is not hard to show that realcompactness and almost realcompactness are equivalent. Thus $X$ is almost realcompact if and only if $E(X)$ is realcompact [5].

This is important for the following reason: suppose $X$ is almost realcompact but not realcompact, e.g., the Dieudonné plank [37, p.

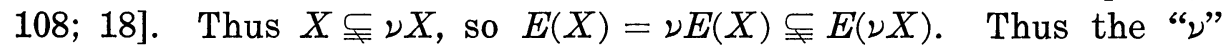
analogue of the " $\beta$ " identity $\beta E(X)=E(\beta X)$ fails. Also, if $p \in \nu X-X$, then $p$ gives rise to a $\sigma$-additive measure $\delta(p)$ on $X$ (explicitly, $\delta(p)(Z)=1$ or 0 according as $p \in \mathrm{cl}_{\beta X} Z$ or not), but there is no obvious candidate for a $\sigma$-additive measure on $E(X)$ which is a preimage of $\delta(p)$. This is the first hint that interesting measuretheoretic pathology may arise when we consider the relation between $X$ and $E(X)$.

Now we turn to one-way implications between $X$ and $E(X)$.

Theorem 2. If $X$ has $P$, then $E(X)$ has $P$, but not conversely: (a) realcompact (b) topologically complete (complete in the finest compatible uniform structure) (c) measure-compact $\left(M_{\sigma}=M_{\tau}\right)$ (d) strongly measure-compact $\left(M_{\sigma}=M_{t}\right)$.

Proof. All of these follow from a lemma of Herrlich and van der Slot [14]: If $f: S \rightarrow T$ is continuous and onto, and $A \subset S, B \subset T$ with $f^{-1}(B)=A$, then $A$ is homeomorphic to a closed subspace of $S \times B$. Merely let $S=E(\beta X), T=\beta X, f=\bar{\kappa}, B=X, A=E(X)$. Now use the fact that each of the four properties is preserved by closed subsets and products with compact spaces (see [29] and [30]).

The Dieudonné plank is a counter-example to all four converses (see §3). However, we will show (Theorem 6, Corollaries) that if $X$ is weak $c b$, then properties (c) and (d) can safely be transferred to Theorem 1; it is well-known [5] that this is true for (a).

THeOREM 3. If $E(X)$ is normal or collectionwise normal, then so is $X$.

Proof. This is not difficult to show. The space $\left[0, \omega_{1}\right)$ is a counter-example to both converses [45]. 
3. Spaces of measures. The maps $\kappa: E(X) \rightarrow X$ and $\bar{\kappa}: \beta E(X) \rightarrow$ $\beta X$ induce linear maps $\kappa^{*}: M(E(X)) \rightarrow M(X)$ and $\bar{\kappa}^{*}: M(\beta E(X)) \rightarrow$ $M(\beta X)$ defined by, for example, $\kappa^{*} \mu(f)=\mu\left(f^{\circ} \kappa\right)$, for each $f \in C^{*}(X)$. In view of the natural identification between $M(X)$ and $M(\beta X)$, and $M(E(X))$ and $M(\beta E(X)), \kappa^{*}$ and $\bar{\kappa}^{*}$ are essentially the same map.

A word of caution is in order concerning the interpretation of functionals as measures (see $\S 3$ of [30]). If $\mu \in M_{\sigma}(E(X))$, then $\kappa^{*} \mu(B)=\mu\left(\kappa^{-1}(B)\right)$, for each Baire set $B$ in $X$. Unfortunately, this desirable result may fail if $\mu$ is only finitely-additive (see $\S 4$ ). Also, we shall think of members of $M(\beta E(X))$ and $M(\beta X)$ as compactregular Borel measures, and then $\bar{\kappa}^{*} \mu(B)=\mu\left(\bar{\kappa}^{-1}(B)\right)$ for each Borel set $B$ in $\beta X$.

Now let $M_{z}$ denote $M_{t}, M_{\tau}$, or $M_{\sigma}$. It is known [30, Th. 3.1] that $\kappa^{*}\left(M_{z}(E(X))\right) \subset M_{z}(X)$, and we are concerned with the question: Is $\kappa^{*-1}\left(M_{z}(X)\right)=M_{z}(E(X))$ ? Unfortunately, the answer is almost always no, and for a very trivial reason: if, say, $x \in \beta X-X$, and $p, q$ are distinct members of $\bar{\kappa}^{-1}(x) \subset \beta E(X)-E(X)$, then $\mu=\delta(p)-$ $\delta(q)$ (a difference of point functionals) is not $\tau$-additive, yet $\kappa^{*} \mu=0$. To avoid this difficulty, we shall be concerned only with nonnegative measures, and regard $\kappa^{*}$ as a map from $M^{+}(E(X))$ to $M^{+}(X)$.

Lemma. $\kappa^{*}$ is a surjection.

Proof. Since $f \rightarrow f^{\circ} \kappa$ is an isometric embedding of $C^{*}(X)$ into $C^{*}(E(X))$, the Hahn-Banach theorem tells us that if $\mu \in M^{+}(X), \exists \lambda \in$ $M(E(X))$ with $\|\lambda\|=\|\mu\|$ and $\lambda\left(f^{\circ} \kappa\right)=\mu(f) \forall f \in C^{*}(X)$. A standard argument shows that $\lambda$ is nonnegative, so $\lambda \in M^{+}(E(X))$ and $\kappa^{*} \lambda=\mu$.

THEOREM 4 . (a) $\kappa^{*-1}\left(M_{t}^{+}(X)\right)=M_{t}^{+}(E(X)) ; \quad$ (b) $\kappa^{*-1}\left(M_{\tau}^{+}(X)\right)=$ $M_{\tau}^{+}(E(X))$.

Proof. Let $\mu \in M_{z}^{+}(X)$, where $z=t$ or $\tau$. We may think of $\mu$ as a regular Borel measure on $\beta X$. Let $\nu$ be a regular Borel measure on $\beta E(X)$ with $\bar{\kappa}^{*} \nu=\mu$.

(a) $z=t$ : There is a sequence $\left(K_{n}\right)$ of compact subsets of $X$ with $\mu\left(\beta X-K_{n}\right)<1 / n \forall n$. Then each $\bar{\kappa}^{-1}\left(K_{n}\right)$ is a compact subset of $E(X)$, and $\nu\left(\beta E(X)-\bar{\kappa}^{-1}\left(K_{n}\right)\right)<1 / n$, so $\nu$ corresponds to a tight measure on $E(X)$.

(b) $z=\tau$ : We have $\mu(L)=0$ for all compact $L \subset \beta X-X[19$, Th. 2.4]. Then if $Q$ is a compact subset of $\beta E(X)-E(X), \bar{\kappa}(Q)$ is a compact subset of $\beta X-X$, so $\nu(Q) \leqq \nu\left(\bar{\kappa}^{-1} \bar{\kappa} Q\right)=\mu(\bar{\kappa} Q)=0$. Hence, by an appeal to the same reference, $\nu$ corresponds to a $\tau$-additive measure on $\beta E(X)$. 
Corollary. $X$ satisfies the condition $M_{t}=M_{\tau}$ if and only if $E(X)$ does.

Other proofs of these results can be found, at least implicitly, in the work of Bauer [3,4] and Topsoe [41]; these proofs rely on nothing more than the fact that $\kappa$ is a perfect map. See also [20].

It would be nice if the argument of Theorem 4(b) also worked for $M_{\sigma}$. Unfortunately it does not: there is no reason to suppose that if $Z$ is a zero-set in $\beta E(X)-E(X)$, then $\bar{\kappa}(Z)$ is a zero-set in $\beta X-X$. However, we can make the following observation: Suppose $X$ has the property

$\left(^{*}\right)$ If $Z$ is a zero-set of $\beta E(X)$, with $Z \subset \beta E(X)-E(X)$, then there is a zero-set $W$ of $\beta X$ with $\bar{\kappa}(Z) \subset W \subset \beta X-X$.

Then it will be true that $\kappa^{*-1}\left(M_{\sigma}^{+}(X)\right)=M_{\sigma}^{+}(E(X))$. The technique of Theorem 4(b), combined with Theorem 2.1 of [19], yields the result, since $\nu(Z) \leqq \nu\left(\bar{\kappa}^{-1} \bar{\kappa} Z\right) \leqq \nu\left(\bar{\kappa}^{-1}(W)\right)=\mu(W)=0$.

We will show that $X$ has $\left(^{*}\right)$ if and only if $X$ is a weak $c b$ space. This concept was introduced by Mack and Johnson [25]; additional details can be found in the book by Alo and Shapiro [1]. For convenience we list several characterizations here.

Lemma [25]. $X$ is a weak cb space if and only if (1) every lacally bounded lower semicontinuous function is bounded above by a continuous function. (2) If $\left(F_{n}\right)$ is a decreasing sequence of regular closed subsets of $X$ with empty intersection, there is a decreasing sequence $\left(Z_{n}\right)$ of zero-sets with $Z_{n} \supset F_{n} \forall n$ and $\bigcap_{1}^{\infty} Z_{n}=\varnothing$. (3) Every countable increasing regular open cover of $X$ has a locally finite partition of unity subordinate to it.

There is a related concept, due to Horne [15], of a $c b$-space. Characterizations of $c b$-spaces may be obtained in the preceding lemma by deleting "lower semicontinuous" from (1) and "regular" from (2) and (3); see [24]. A space is $c b$ is and only if it is weak $c b$ and countably paracompact. Other relationships with standard topological properties are summarized in the following diagram:

normal and countably paracompact

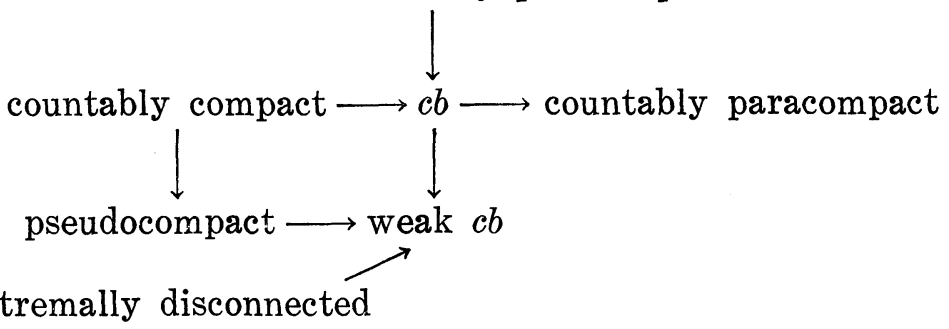


A realcompact, even a measure-compact space need not be weak $c b$ (Michael's product space [27]). Conversely, a weak $c b$ space need not be realcompact (any pseudocompact, noncompact space). The space $R^{c}$ is weak $c b$ and realcompact, but not measure-compact.

THeOrem 5. $X$ has $\left(^{*}\right)$ if and only if $X$ is a weak cb space.

Proof. (a) Suppose $X$ has (*). We prove (3) of the preceding lemma. Let $\left(U_{n}\right)_{1}^{\infty}$ be an increasing regular open cover of $X$. Let $F_{n}=X-U_{n}$, and let $L=\bigcap_{1}^{\infty} \operatorname{cl}_{\beta X} F_{n}$. Then $L$ is a compact subset of $\beta X-X$. Each $F_{n}$ is regular closed in $X$, so $\operatorname{cl}_{\beta X} F_{n}$ is regular closed in $\beta X$, and $\bar{\lambda}\left(\operatorname{cl}_{\beta X} F_{n}\right)=C_{n}$ is a clopen subset of $E(\beta X)$.

Let $Z=\cap_{1}^{\infty} C_{n}$. Then $Z$ is a zero-set in $E(\beta X)$, and certainly $\bar{\kappa}(Z) \subset L$; indeed, using the technique of [48, Lemma 2.4] one can show that $\bar{\kappa}(Z)=L$. Then $Z \subset \bar{\kappa}^{-1}(L) \subset \beta E(X)-E(X)$, so, from $\left({ }^{*}\right)$, there is a zero-set $W$ of $\beta X$ with $\bar{\kappa}(Z)=L \subset W \subset \beta X-X$. Then $T=\beta X-W$ is $\sigma$-compact locally compact, and $\bigcap_{1}^{\infty} \operatorname{cl}_{T} F_{n}=\varnothing$, so $\left\{T-\mathrm{cl}_{T} F_{n}\right\}$ is an open cover of $T$. Choose a locally finite (in $T$ ) partition of unity $\left(f_{\alpha}\right)$ subordinate to this cover. Then if $g_{\alpha}=f_{\alpha} \mid X$, $\left(g_{\alpha}\right)$ is a locally finite partition of unity in $X$, subordinate to $\left\{\left(T-\mathrm{cl}_{T} F_{n}\right) \cap X\right\}=\left\{U_{n}\right\}$. Thus $X$ is a weak $c b$ space.

(b) Assume $X$ is a weak $c b$ space, and let $Z$ be a zero-set of $\beta E(X)$, with $Z \subset \beta E(X)-E(X)$. There is a decreasing sequence $\left(C_{n}\right)$ of clopen subsets of $\beta E(X)$ with $\bigcap_{1}^{\infty} C_{n}=Z$. Let $\bar{\kappa}\left(C_{n}\right)=D_{n}$ and $D=\bigcap_{1}^{\infty} D_{n}$. Then each $D_{n}$ is regular closed in $\beta X$, and (as in part (a)) $\bar{\kappa}(Z)=D \subset \beta X-X$.

Now let $F_{n}=D_{n} \cap X$; then $\left(F_{n}\right)$ is a decreasing sequence in $R C(X)$ with empty intersection. From (2) of the lemma, there is a decreasing sequence $\left(Z_{n}\right)$ of zero-sets with $Z_{n} \supset F_{n} \forall n$ and $\bigcap_{1}^{\infty} Z_{n}=\varnothing$. Let $Z_{n}=f_{n}^{-1}(0), f_{n} \in C^{*}(X)$. If $\bar{f}_{n}$ is the extension to $\beta X$, certainly $\operatorname{cl}_{\beta X} Z_{n} \subset W_{n}=\bar{f}_{n}^{-1}(0)$. Let $W=\bigcap_{1}^{\infty} W_{n}$. Then $W$ is a zero-set of $\beta X$, and $W \subset \beta X-X$. Hence $\bar{\kappa}(Z)=D=\cap \operatorname{cl}_{\beta X} F_{n} \subset \cap \operatorname{cl}_{\beta X} Z_{n} \subset$ $\bigcap_{1}^{\infty} W_{n}=W \subset \beta X-X$. This shows that $\left(^{*}\right)$ holds.

Recall the definitions of the strong and weak lifting properties given in the introduction.

THEOREM 6. If $X$ is measure-compact or weak cb, then $X$ has the SLP.

Proof. If $X$ is measure-compact, the result is immediate from Theorem $4 \mathrm{~b}$. If $X$ is weak $c b$, then Theorem 5 and the remarks following the definition of $\left(^{*}\right)$ yield the result.

Corollary 1. If $X$ is weak $\mathrm{cb}$, then $X$ is measure-compact if and only if $E(X)$ is measure-compact. 
Proof. Theorems 2c and 6.

CoROllary 2. If $X$ is weak $\mathrm{cb}$, then $X$ is strongly measurecompact if and only if $E(X)$ is strongly measure-compact.

Proof. Theorem 2d, Theorem 4 (Corollary), and Theorem 6.

Several remarks are in order here.

(1) If $X$ is weak $c b$, so is $\nu X$ [25]; thus $\nu X$ is measure-compact if and only if $E(\nu X)$ is measure-compact.

(2) Corollary 1 could be phrased: If $X$ has the WLP, then $X$ is measure-compact if and only if $E(X)$ is measure-compact. The following is also valid: if $E(X)$ is measure-compact, then $X$ has the WLP if and only if $X$ has the SLP. For if $\mu \in M_{\sigma}^{+}(X)$ has a single $\sigma$-additive pre-image $\nu$, then $\nu$ is $\tau$-additive, hence so is $\mu$, and therefore every pre-image of $\mu$ is $\tau$-additive, by Theorem $4 \mathrm{~b}$. As we shall see (Example 1 below), it is possible for $E(X)$ to be (strongly) measure-compact without $X$ possessing the WLP.

(3) Let $X$ be the Sorgenfrey plane. Then $X$ is realcompact and weak $c b$ [25], but not measure-compact [28]. Hence $E(X)$ is $a$ realcompact extremally disconnected space which is not measurecompact.

EXAmPle 1. A space which fails the WLP.

Let $D$ be the Dieudonné plank. Thus $D=\left[0, \omega_{1}\right] \times\left[0, \omega_{0}\right]-$ $\left\{\left(\omega_{1}, \omega_{0}\right)\right\}$. Here $\left[0, \omega_{0}\right]$ is topologized as usual (order topology), while $\left[0, \omega_{1}\right]$ is given the topology in which each $\alpha<\omega_{1}$ is isolated, and $\left\{\left(\beta, \omega_{1}\right]\right\}_{\beta<\omega_{1}}$ is a base of neighborhoods of $\omega_{1}$. $D$ has the subspace topology arising from the product topology. Let $p=\left(\omega_{1}, \omega_{0}\right)$.

Kato [18] has shown that (1) $D$ is almost realcompact; (2) $D$ is not realcompact and (3) $\nu D=D \cup\{p\}$ is Lindelöf.

We will show that (A) $\mu=\delta(p)$ is a $\sigma$-additive measure on $D$ with no pre-image in $M_{\sigma}^{+}(E(D))$ and (B) $E(D)$ is strongly measurecompact, although $D$ is not even realcompact.

In order to do this, we follow Kato in introducing an auxiliary space $\widetilde{D}$. Let $G=\left[0, \omega_{1}\right) \times\left[0, \omega_{0}\right] \subset D$, and let $\widetilde{G}$ be a copy of $G$. Then the embedding $i: \widetilde{G} \rightarrow G \subset D$ extends to a map $\psi: \beta \widetilde{G} \rightarrow \beta D$. Since $G$ is dense in $D, \psi$ is onto. By [11, Lemma 6.11], $\psi$ maps $\beta \widetilde{G}-\widetilde{G}$ onto $\beta D-G$. Now let $\widetilde{D}=\psi^{-1}(D)$ and $\Phi=\psi \mid \widetilde{D}$. Then $\widetilde{G} \subset \widetilde{D}$, and $\Phi$ maps $\widetilde{D}-\widetilde{G}$ onto $D-G$ (the right edge of $D$ ).

Now $\Phi$ is a perfect map, since it is the restriction of $\psi$ to a complete inverse image; since $\widetilde{D}$ contains a dense set of isolated points, $\Phi$ is also irreducible. Consider the diagram 


$$
E(\widetilde{D}) \stackrel{\tilde{\kappa}}{\longrightarrow} \widetilde{D} \stackrel{\Phi}{\longrightarrow} D
$$

Since $\tilde{\kappa}$ and $\Phi$ are perfect and irreducible, so is $\Phi \circ \tilde{\kappa}$; we deduce that $E(D)=E(\widetilde{D})$ and $\kappa=\Phi \circ \tilde{\kappa}$.

Proof of (A). Suppose $\exists \lambda \in M_{\sigma}^{+}(E(D))$ with $\kappa^{*} \lambda=\mu$. Let $\nu=$ $\tilde{\kappa}^{*}(\lambda) \in M_{o}^{+}(\widetilde{D})$; then $\Phi^{*} \nu=\mu$. Thus to establish (A), it is enough to show that no such $\nu$ can be found.

Suppose there were such a $\nu$. Let $T=\left\{\left(\alpha, \omega_{0}\right): \alpha<\omega_{1}\right\}$ be the top edge of $D$, and let $\widetilde{T}=\Phi^{-1}(T) \subset \widetilde{D}$. Since $T$ is a zero-set of $D$, with $\mu(T)=\mu(D)=1$, we have $\widetilde{T}$ a zero-set of $\widetilde{D}$, with $\nu(\widetilde{T})=$ $\nu(\widetilde{D})=1$. Since $T \subset G, \widetilde{T}$ and $T$ are homeomorphic under $\Phi$; each is a discrete space of cardinal $\boldsymbol{\aleph}_{1}$. But there is a critical distinction between them: $T$ is not $C^{*}$-embedded in $D$, but $\widetilde{T}$ is $C^{*}$-embedded in $\widetilde{D}$ (because it is $C^{*}$-embedded in $\widetilde{G}$, hence in $\beta \widetilde{G}$ ). It follows that every subset of $\widetilde{T}$ is a zero-set of $\widetilde{D}$. Thus $\nu$ is defined on all subsets of a set of cardinal $\aleph_{1}$, has total mass 1 , and assigns measure 0 to all singletons. This contradicts a well-known result of Ulam [42]. Hence no such $\nu$ can exist.

Proof of (B). The only $\tau$-additive measures on $D$ are of the form $\sum_{1}^{\infty} c_{n} \delta\left(x_{n}\right),\left(x_{n}\right) \in D, \sum_{1}^{\infty}\left|c_{n}\right|<\infty$; hence $M_{\tau}(D)=M_{t}(D)$, and so, by the Corollary to Theorem $4, E(D)$ has the same property. It remains to show that $E(D)$ is measure-compact, and, using the same technique as in the proof of Theorem 2, it is enough to show that $\widetilde{D}$ is measure-compact. We use the criterion of Moran [28, Th. 2.1].

Suppose $\mu \in M_{\sigma}^{+}(\widetilde{D})$ and $\mu$ has empty support. Let $L_{n}=\{(\alpha, n)$ : $\left.\alpha \leqq \omega_{1}\right\}$ for each $n$. Then $L_{n}$ is a Lindelöf, clopen subset of $D$, and so (Theorem $1(\mathrm{c})) \Phi^{-1}\left(L_{n}\right)$ is a Lindelöf clopen subset of $\widetilde{D}$. Thus $\mu \mid \Phi^{-1}\left(L_{n}\right) \equiv 0 \forall n$. Also, as in (A), $\mu \mid \widetilde{T}$ is defined on all subsets and vanishes on all singletons. Thus $\mu$ is the 0 measure, to complete the proof.

REMARK. Let $X$ be any almost realcompact, nonrealcompact space. Then $X$ fails the SLP. Indeed choose $x_{0} \in \nu X-X$, and note that, while $x_{0}$ has pre-images in $\beta E(X)$, none can lie in $\nu E(X)=E(X)$. Thus $\mu=\delta\left(x_{0}\right) \in M_{o}^{+}(X)$, but none of the point-mass pre-images of $\mu$ is $\sigma$-additive, so $X$ does not have the SLP.

We conjecture that such a $\mu$ has no $\sigma$-additive pre-image of any kind, so that such an $X$ actually fails the WLP, as we have just seen for the special case $X=D$.

EXAMPLE 2. A space which has the WLP but fails the SLP. 
Let $X$ be the space obtained by joining the Dieudonne plank $D$ and the Tychonoff plank $T$ along the edge $\left\{\left(\omega_{1}, n\right): n \in N\right\}$. Then $X$ is completely regular Hausdorff, and $D$ and $T$ are regular closed subsets of $X$, each the Boolean complement of the other. The ultrafilters of regular closed sets thus divide nicely into two classes, those which contain $D$ and those which contain $T$, and in fact $E(X)$ is the topological sum of $E(D)$ and $E(T)$ (cf. [48, Lemma 2.6]).

Let $p=\left(\omega_{1}, \omega_{0}\right)$; then $X \cup\{p\}$ is Lindelöf, and contains $X$ as a dense, $C$-embedded subspace, so $\nu X=X \cup\{p\}$. Then $\mu=\delta(p) \epsilon$ $M_{\sigma}^{+}(X)$, and it is easy to see that scalar multiples of $\mu$ are the only purely $\sigma$-additive measures on $X$. Thus it is enough to show that $\kappa^{*-1}(\mu)$ contains both $\sigma$-additive and non $\sigma$-additive measures. Now $D$ and $T$ are both $\mu$-thick $\left(\mu^{*} D=\mu^{*} T=1\right)$ and $C^{*}$-embedded in $X$, so $\mu$ induces measures $\mu_{1} \in M_{\sigma}^{+}(D)$ and $\mu_{2} \in M_{\sigma}^{+}(T)$; each is simply the "point mass at $p$ " in its own setting. By the lemma at the beginning of this section, $\exists \lambda_{1} \in M^{+}(E(D))$ with $\kappa_{D}^{*} \lambda_{1}=\mu_{1}$, and $\exists \lambda_{2} \in M^{+}(E(T))$ with $\kappa_{T}^{*} \lambda_{2}=\mu_{2}$. By Example $1 \lambda_{1}$ cannot be $\sigma$-additive. Since $T$ is pseudocompact, so is $E(T)$, and therefore $\lambda_{2}$ must be $\sigma$-additive [43, p. 172]. Since each can be viewed as a pre-image of $\mu$ in $M^{+}(E(T))$, the proof is complete.

Isawata has defined a space $X$ to be a weak $c b^{*}$ space if whenever $\left(F_{n}\right)$ is a sequence of regular closed sets in $X$ with $\bigcap_{1}^{\infty} F_{n}=\varnothing$ then $\bigcap_{1}^{\infty} \mathrm{cl}_{\nu X} F_{n}=\varnothing$. Hardy and Woods [49] showed that $X$ is weak $c b^{*}$ if and only if $E(\nu X)=\nu E(X)$. An almost realcompact, nonrealcompact space is never weak $c b^{*}$. At this point we summarize our findings in a diagram:

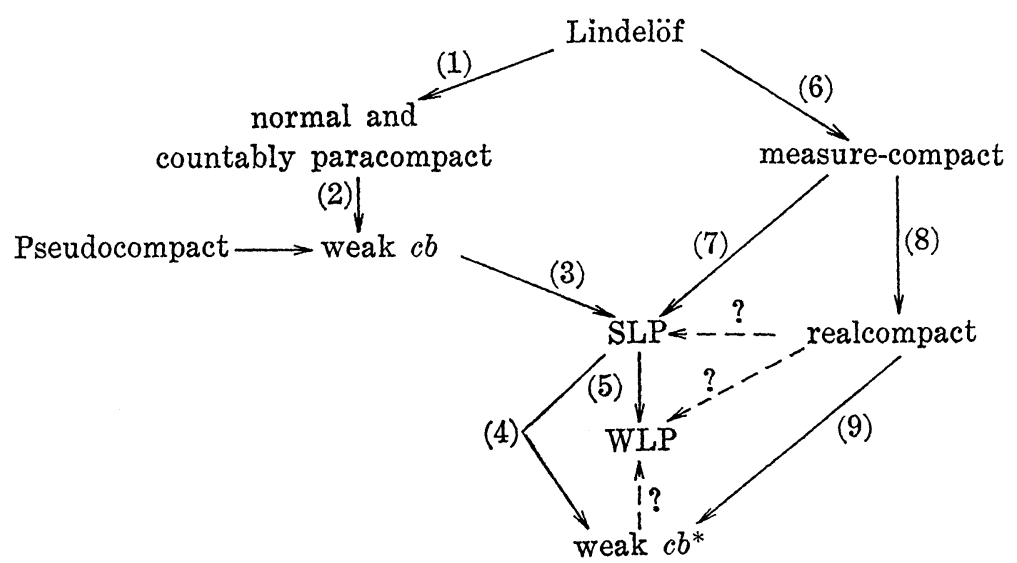

REMARKs. (1), (2), (6), and (8) are well-known, and each converse is false. (3) and (7) are proved in Theorem 6; the disjoint 
union of Michael's product space and the Sorgenfrey plane has the SLP, but is neither weak $c b$ nor measure-compact. (5) is trivial; Example 2 shows that the converse fails. (9) is equally trivial, and the converse fails (any pseudocompact, noncompact space). (4) can be shown as follows: it is always true that $\nu E(X) \subset E(\nu X)$. If $p \in$ $E(\nu X)$, then $\bar{\kappa}(p) \in \nu X$, and so $\delta(\bar{\kappa}(p))=\kappa^{*}(\delta(p)) \in M_{\sigma}^{+}(X)$. Hence (from the SLP) $\delta(p) \in M_{\sigma}^{+}(E(X))$, i.e., $p \in \nu E(X)$.

The SLP (and hence the WLP) do not imply realcompactness (any pseudocompact, noncompact space). The space of Example 2 has the WLP, but is not weak $c b^{*}$. To see this, note that $E(\nu X)$ is Lindelöf, since $\nu X$ has that property. However, $\nu E(X)$ is the topological sum of $\nu E(D)=E(D)$ and $\nu E(T)=\beta E(T)$. Since $D$ is not Lindelöf, neither is $E(D)$, and so $\nu E(X) \varsubsetneqq E(\nu X)$.

Since a zero-set of $\beta X-X$ must actually lie in $\beta X-\nu X$, there is a natural identification of $M_{\sigma}(X)$ and $M_{\sigma}(\nu X)$. This yields the following proposition; we omit the proof.

THEOREM 7(a). If $X$ has the WLP (or SLP), so does $\nu X$. $X$ is weak $\mathrm{cb}^{*}$, and $\nu X$ has the WLP (or SLP), then so does $X$.

In view of these results, the principal open question appears to be: Does realcompactness imply the WLP or the SLP? We know that the WLP fails for the Dieudonné plank, but this is basically a topological pathology: there is a point of $\nu X-X$ with no pre-image in $\nu E(X)-E(X)$, simply because the latter set is empty. Failure of the WLP for a realcompact space would be a true measure-theoretic pathology. Such an example would be quite interesting; we mention a possible candidate below.

Example 3. Let $X=[0,1]$, endowed with the density topology $\mathscr{T}_{d}$. A subset $E$ of $[0,1]$ is open in this topology if and only if $E$ is Lebesgue measurable and has density one at each of its points; see [34] and [40] for details. We shall need the the following facts: (1) $\mathscr{T}_{d}$ is finer than the usual topology $\mathscr{T}_{0}$ on $X$; $(2)\left(X, \mathscr{T}_{d}\right)$ is realcompact; (3) Every $\mathscr{T}_{d}$-continuous function is Baire class 1; hence the $\mathscr{T}_{d}$-Baire sets are precisely the $\mathscr{T}_{0}$-Borel sets; (4) Every set of Lebesgue measure 0 is $\mathscr{T}_{d}$-closed and discrete; (5) Let $\mathscr{L}$ be the $\sigma$-algebra of Lebesgue measurable sets, $m=$ Lebesgue measure. Then $\beta E(X)$ is the Stone space of the reduced measure algebra $\mathscr{L} / m^{-1}(0)$ (henceforth $E(X)$ stands for $E\left(X, \mathscr{T}_{d}\right)$ ).

Now let $C$ be the Cantor set, and, thinking of $C$ as $2^{N}$, let $\mu$ denote Haar measure on the Borel sets of $C$. From (3), we may think of $\mu$ as a member of $M_{\sigma}^{+}\left(X, \mathscr{T}_{d}\right)$. But it is easy to see, using 
(4), that spt $\mu=\left\{x \in X\right.$ : if $x \in U\left(\mathscr{T}_{d}\right.$-open), then $\left.\mu U>0\right\}$ is empty, so $\mu$ is not $\tau$-additive; hence $\left(X, \mathscr{T}_{d}\right)$ is not measure-compact.

We now ask: does there exist $\lambda \in M_{o}^{+}(E(X))$ with $\kappa^{*}(\lambda)=\mu$ ? Intuitively it seems unlikely, since the measure algebra construction suppresses sets like $C$. However, we now show that if $\mathscr{L}$ admits a Borel lifting of a certain type, then $\left(X, \mathscr{T}_{d}\right)$ has the WLP, so that such a $\lambda$ does exist.

For a discussion of the theory of lifting, see [16] or [26]. If $A \in \mathscr{L}$, let $\theta(A)=\{x \in X$ : the density of $A$ at $x$ is 1$\}$.

THEOREM 8. Suppose there is a lifting $\mathfrak{l}: \mathscr{L} \rightarrow \mathscr{L}$ such that (a) $\theta(A) \subset \mathfrak{l}(A) \forall A \in \mathscr{L}$ and (b) each set $\mathfrak{I}(A)$ is a $\mathscr{T}_{0}$-Borel set. Then $\left(X, \mathscr{T}_{d}\right)$ has the WLP.

Proof. The lifting $\mathfrak{l}$ gives rise to a lifting topology $\mathscr{T}_{\mathfrak{l}}$ on $X$ for which $\{\mathfrak{I}(A): A \in \mathscr{L}\}$ is a base. Condition (a) implies that $\mathscr{T}_{0} \subset$ $\mathscr{T}_{d} \subset \mathscr{T}_{1}$ on $X$, and condition (b) implies that each $\mathscr{T}_{\mathfrak{r}}$-continuous function is $\mathscr{T}_{0}$-Borel measurable; hence the $\mathscr{T}_{\mathfrak{r}}$-Baire sets coincide with the $\mathscr{T}_{0}$-Borel sets and the $\mathscr{T}_{d}$-Baire sets.

From [16, pp. 58-61] we can deduce that there is a dense subspace $Y$ of $E(X)$ which is homeomorphic to $\left(X, \mathscr{T}_{1}\right)$. $Y$ consists of exactly one point from each set $\kappa^{-1}(x)$; explicitly, $x$ corresponds to the ultrafilter $p=\left\{F \in R C\left(X, \mathscr{T}_{d}\right): x \in \mathfrak{I}(F)\right\}$.

Now let $\mu \in M_{\sigma}^{+}\left(X, \mathscr{T}_{d}\right)$. There is a corresponding Baire measure $\hat{\mu}$ on $Y \subset E(X)$. Define $\gamma$ on the Baire sets of $E(X)$ by $\gamma(B)=$ $\widehat{\mu}(B \cap Y)$. Then $\gamma \in M_{o}^{+}(E(X))$, and if $D$ is a Baire set in $\left(X, \mathscr{T}_{d}\right)$, $\gamma\left(\kappa^{-1}(D)\right)=\hat{\mu}\left(\kappa^{-1}(D) \cap Y\right)=\mu(D)$. Hence $\kappa^{*} \gamma=\mu$, and so $\left(X, \mathscr{T}_{d}\right)$ has the WLP.

Thanks are due to Dennis Sentilles for some very helpful ideas relative to this proof.

Assuming the continuum hypothesis, Siegfried Graf has shown [52, Th. 9.2] that a lifting satisfying the conditions of Theorem 8 exists (see also [23] and [44]). Without the continuum hypothesis, however, the question of existence of such a lifting seems to be open.

4. Extension of measures. Up to this point we have treated $M^{+}(E(X))$ and $M^{+}(X)$ as distinct collections of measures connected by the map $\kappa^{*}$. Here is another point of view: if $\mu \in M^{+}(X)$, then $\mu$ induces a set function $\lambda$ on $\left\{\kappa^{-1}(B): B\right.$ a Baire set in $\left.X\right\}$ in the obvious way: $\lambda\left(\kappa^{-1}(B)\right)=\mu(B)$. The question then becomes: does $\lambda$ have an extension to a zero-set regular measure $\nu$ defined on all Baire sets of $E(X)$ ? Call such a $\nu$ a measure extension of $\mu$.

We consider this problem in the context of a recent paper on 
extensions of measures by Sultan [39]. We record some relevant terminology. A lattice $\mathscr{L}$ of subsets of a set $X$ is called a paving. $\mathscr{L}$ is a delta paving if $\mathscr{L}$ is closed under countable intersections; $\mathscr{L}$ is a normal paving if disjoint members of $\mathscr{L}$ are contained in disjoint complements of members of $\mathscr{L}$. If $\mathscr{L}_{1} \subset \mathscr{L}_{2}$ are two lattices of subsets of $X$, then $\mathscr{L}_{1}$ semiseparates $\mathscr{L}_{2}$ if whenever $A \in \mathscr{L}_{1}$, $B \in \mathscr{L}_{2}$, and $A \cap B=\varnothing$, there exists a $C \in \mathscr{L}_{1}$ such that $B \subset C$ and $A \cap C=\varnothing$. Finally, $\mathscr{L}_{2}$ is $\mathscr{L}_{1}$ c.b. if $B_{n} \downarrow \varnothing$ in $\mathscr{L}_{2}$ implies there exist $\left(A_{n}\right)$ in $\mathscr{L}_{1}$ with $B_{n} \subset A_{n} \downarrow \varnothing$.

Now let $\mathscr{L}_{1}=\left\{\kappa^{-1}(Z): Z\right.$ a zero-set of $\left.X\right\}$, and let $\mathscr{L}_{2}=\{W: W$ a zero-set of $E(X)$. Then $\mathscr{L}_{1} \subset \mathscr{L}_{2}$, and each is a delta-normal paving of subsets of $E(X) . \quad M^{+}(X)$ corresponds to $M R\left(\mathscr{L}_{1}\right)$, and $M^{+}(E(X))$ corresponds to $M R\left(\mathscr{L}_{2}\right)$.

We now give topological characterizations of the semiseparation and c.b. properties. Mack [54] has termed a space weakly $\delta$-normally separated if each regular closed set and zero-set disjoint from it are completely separated. He has shown that every weak $c b$ space has this property.

Lemma. Suppose $X$ is any completely regular space, and $F, H$ are disjoint closed subsets of $X$, with $F$ a zero-set and $H$ the intersection of a decreasing sequence of regular closed sets (an $R C_{\dot{o}}$ ). Then there is a regular closed set $D$ with $H \subset D$ and $F \cap D=\varnothing$.

Proof. Let $H=\bigcap_{1}^{\infty} H_{n}$, where each $H_{n}$ is regular closed and $H_{n} \supset H_{n+1} \forall n$. Let $A=\kappa^{-1}(F), B=\bigcap_{1}^{\infty} \lambda\left(H_{n}\right)$. Then $A$ and $B$ are disjoint zero-sets in $E(X)$, and so there is a clopen set $C$ with $B \subset C$ and $A \cap C=\varnothing$. Then $D=\kappa(C)$ is the desired regular closed set.

THEOREM 9. $\mathscr{L}_{1}$ semiseparates $\mathscr{L}_{2}$ [39] if and only if $X$ is weakly $\delta$-normally separated.

Proof. Assume the topological condition, and let $A \in \mathscr{L}_{1}, B \in \mathscr{L}_{2}$ with $A \cap B=\varnothing$. Let $A=\kappa^{-1}(F)$, where $F$ is a zero-set of $X$. Now $B$ is a countable intersection of clopen subsets of $E(X)$, and so $\kappa(B)=H$ is an $R C_{\delta}$ in $X$. From the hypothesis and the lemma, there is an $f \in C^{*}(X), f|F \equiv 1, f| H \equiv 0$. Then if $C=\kappa^{-1}(Z(f))$, we have $B \subset C$ and $A \cap C=\varnothing$.

Conversely, suppose $\mathscr{L}_{1}$ semiseparates $\mathscr{L}_{2}$. Then for disjoint $F$ and $H$ in $X, F$ a zero-set and $H$ regular closed, $\kappa^{-1}(F) \in \mathscr{L}_{1}$ and $\lambda(H) \in \mathscr{L}_{2}$ are disjoint, hence there is a zero-set $Z$ of $X$ with $\lambda(H) €$ $\kappa^{-1}(Z)$ and $\kappa^{-1}(Z) \cap \kappa^{-1}(F)=\varnothing$. It follows that $F$ and $H$ are completely separated. 
COROLLARY. The following are equivalent:

(1) $X$ is weakly $\delta$-normally separated.

(2) if $\mu \in M^{+}(X)$, and $\nu$ is any measure in $M^{+}(E(X))$ with $\kappa^{*} \nu=\mu$, then $\nu$ is a measure extension of $\mu$.

(3) if $p \in \beta X$, and $q$ is any point of $\beta E(X)$ with $\bar{\kappa}(q)=p$, then $\delta(q)$ is a measure extension of $\delta(p)$.

Proof. $\quad(1) \Rightarrow(2)$ : Theorem 9, and Lemma 4.4 of [39]. $\quad(2) \Rightarrow$ (3): trivial, since (3) is a special case of $(2) . \quad(3) \Rightarrow(1)$ : Woods [55] has shown that $X$ is weakly $\delta$-normally separated if and only if $\operatorname{cl}_{\beta E(X)} \kappa^{-1}(Z)=\bar{\kappa}^{-1}\left(\operatorname{cl}_{\beta X} Z\right)$ for each zero-set $Z$ of $X$. If (1) fails, choose a $Z_{0}$ for which $\operatorname{cl}_{\beta E(X)} \kappa^{-1}\left(Z_{0}\right) \subsetneq \bar{\kappa}^{-1}\left(\operatorname{cl}_{\beta X} Z_{0}\right)$, and find a point $q$ in the second set, but not the first. Then, with $\bar{\kappa}(q)=p$, we have $\delta(p)\left(Z_{0}\right)=1$, since $p \in \operatorname{cl}_{\beta X} Z_{0}$, but $\delta(q)\left(\kappa^{-1}\left(Z_{0}\right)\right)=0$. Thus $\delta(q)$ is not a measure extension of $\delta(p)$.

ExAMPLE. Referring to Example 2, let $Y$ be the space obtained by deleting from $X$ the top edge of the Tychonoff plank $T$, and let $U$ be the portion of $T$ which remains. Then $Y$ is not weakly $\delta$ normally separated (consider $Z$, the top edge of $D$, and $U$ ). As in Example 2, $\nu Y=Y \cup\{p\}$, so $\delta(p) \in M_{\sigma}^{+}(Y) . E(Y)$ is the topological sum of $E(D)$ and $E(U)$; choose $q \in \beta E(U)$ with $\bar{\kappa}(q)=p$. Then $\delta(p)(Z)=1$, but $\delta(q)\left(\kappa^{-1}(Z)\right) \leqq \delta(q)(E(D))=0$. Thus $\delta(p)$ is a $\sigma$-additive measure with a functional extension $\delta(q)$ which is not a measure extension; $\delta(q)$ is (necessarily) only finitely-additive.

REMARK. For any $X$ and any $p \in \beta X$, there is always at least one $q \in \beta E(X)$ with $\bar{\kappa}(q)=p$ and $\delta(q)$ a measure extension of $\delta(p)$. Indeed $\left\{\kappa^{-1}(Z): p \in \operatorname{cl}_{\beta X} Z\right\}$ is a $z$-filterbase on $E(X)$; any $z$-ultrafilter which extends it corresponds to such a $q$.

Question. Is it true that for any $X$ and $\mu \in M^{+}(X)$, there is always at least one $\nu \in M^{+}(E(X))$ with $\kappa^{*}(\nu)=\mu$ and $\nu$ a measure extension of $\mu$ ?

THEOREM 10. $\mathscr{L}_{2}$ is $\mathscr{L}_{1}$ c.b. [39] if and only if $X$ is a weak cb space.

Proof. Assume $X$ is weak $\mathrm{cb}$, and let $B_{n} \downarrow \varnothing$ in $\mathscr{L}_{2}$. Let $B_{n}=$ $\bigcap_{i} C_{n, i}$, where each $C_{n, i}$ is clopen, and let $D_{n}=\bigcap_{m, i=1}^{n} C_{m, i}$. Then $D_{n} \downarrow \varnothing$, and so $\kappa\left(D_{n}\right) \downarrow \varnothing$ in $R C(X)$. Choose a sequence $\left(Z_{n}\right)$ of zerosets of $X$ with $Z_{n} \supset \kappa\left(D_{n}\right)$ and $Z_{n} \downarrow \varnothing$. Then with $A_{n}=\kappa^{-1}\left(Z_{n}\right)$, we have $B_{n} \subset A_{n}$ and $A_{n} \downarrow \varnothing$.

The proof of the converse is left to the reader. 
In view of Theorems 9 and 10 and Mack's result that every weak $\mathrm{cb}$ space is weakly $\delta$-normally separated, Theorem 5.1 of [39] yields another proof that every weak cb space has the strong lifting property (Theorem 6).

5. Spaces of functions. As we have remarked, the map $\Phi$ : $C^{*}(X) \rightarrow C^{*}(E(X))$ defined by $\Phi(f)=f^{\circ} \kappa$ is an isometric embedding of $C^{*}(X)$ as a norm-closed subspace of $C^{*}(E(X))$. Since $\kappa$ is closed and onto, it is a quotient map; hence $g \in C^{*}(E(X))$ is a member of $\Phi\left(C^{*}(X)\right)$ if and only if $g \mid \kappa^{-1}(x)$ is constant for each $x \in X$. It follows that $\Phi\left(C^{*}(X)\right)$ is actually a pointwise-closed subspace of $C^{*}(E(X))$.

We now investigate whether $\Phi$ is a topological embedding with respect to the strict topologies $\beta_{0}, \beta$, and $\beta_{1}$ [35] which can be placed on $C^{*}(X)$ and $C^{*}(E(X))$. As might be expected from $\S 3$, the situation is as nice as possible for $\beta_{0}$ and $\beta$, and somewhat complicated for $\beta_{1}$.

THEOREM 11. $\Phi$ is a topological isomorphism of $\left(C^{*}(X), \mathscr{Y}^{-}\right)$ onto a closed subspace of $\left(C^{*}(E(X)), \mathscr{T}\right)$ for $\mathscr{T}=\beta_{0}$ or $\beta$.

Proof. It is known [30] that $\Phi$ is $\mathscr{S}$-continuous in either case. $\left(\mathscr{S}=\beta_{0}\right)$ Let $U$ be a $\beta_{0}$-neighborhood of 0 in $C^{*}(X)$. We may assume [35] that $U=\bigcap_{1}^{\infty}\left\{f \mid\|f\|_{H_{i}} \leqq a_{i}\right\}$, where each $H_{i}$ is a compact subset of $X$ and $0<a_{i} \uparrow \infty$. Let $L_{i}=\kappa^{-1}\left(H_{i}\right)$. Then each $L_{i}$ is compact, and $\Phi(U) \supset \Phi\left(C^{*}(X)\right) \cap \bigcap_{1}^{\infty}\left\{g \mid\|g\|_{L_{i}} \leqq a_{i}\right\}$, so $\Phi$ is open onto its range.

$(\mathscr{T}=\beta)$ Let $U$ be a $\beta$-neighborhood of 0 in $C^{*}(X)$. We may assume [35] that $U=H^{\circ}$, where $H$ is weak ${ }^{*}$-compact subset of $M_{\tau}^{+}(X)$. Now $\kappa^{*-1}(H)=Q$ is weak ${ }^{*}$-compact in $M^{+}(E(X))$, by Alaoglus, theorem, and so, by Theorem $4 \mathrm{~b}, Q$ is a weak*-compact subset of $M_{\tau}^{+}(E(X))$, hence $\beta$-equicontinuous. Thus $\Phi(U) \supset \Phi\left(C^{*}(X)\right) \cap Q^{\circ}$, so $\Phi$ is open onto its range.

We remark that (1) the $\mathscr{T}=\beta_{0}$ proof adapts easily to show that $\Phi$ is an embedding of $C(X)$ in $C(E(X))$ for the compact-open topology; (2) If $C^{*}(E(X))$ is $\beta_{0}$ or $\beta$-complete, so is $C^{*}(X)$. In the $\beta_{0}$-case this says, topologically, that if $E(X)$ is a $k_{R}$-space, (i.e., every real-valued function which is continuous on compact subsets is continuous), then so is $X$. Of course, this is trivial to prove directly. The converse ( $X$ a $k_{R}$-space $\Rightarrow E(X)$ a $k_{R}$-space) seems to be open.

Definition 3. $X$ is $\beta_{1}$-stable if $\Phi$ is a topological isomorphism of $\left(C^{*}(X), \beta_{1}\right)$ onto a closed subspace of $\left(C^{*}(E(X)), \beta_{1}\right)$. 
LeMma [53, p. 156]. If $E$ is a locally solid vector lattice, $F$ is a linear subspace and sublattice of $E$, and $\mu$ is a positive continuous linear functional on $F$, then there is a positive continuous linear functional $\lambda$ on $E$ with $\lambda \mid F=\mu$.

\section{THEOREM 12. $\mathrm{SLP} \Rightarrow \beta_{1}$-stable $\Rightarrow$ WLP.}

Proof. That the SLP $\Rightarrow \beta_{1}$-stable can be proved just as in the $\mathscr{T}=\beta$ case of the previous theorem. Now suppose that $X$ is $\beta_{1}$ stable, and let $\mu \in M_{\sigma}^{+}(X)$. Then $\mu$ can be thought of as a $\beta_{1}$-continuous linear functional on $\Phi\left(C^{*}(X)\right)$, so by the Hahn-Banach theorem, $\exists \lambda \in M_{\sigma}(E(X))$ with $\kappa^{*} \lambda=\mu$. Since $\beta_{1}$ is locally solid, the lemma shows that we can choose $\lambda$ to be nonnegative.

The first implication, at least, of Theorem 12 cannot be reversed.

Example 2 (continued). The join $X$ of the Dieudonne plank $D$ and the Tychonoff plank $T$ is $\beta_{1}$-stable, but fails the SLP.

Proof. We only sketch the argument. It is enough to show that if $H$ is a weak*-compact subset of $M_{o}^{+}(X)$, then there is a weak*-compact subset $Q$ of $M_{\sigma}^{+}(E(X))$ with $\kappa^{*}(Q)=H$. It is convenient to identify $M_{\sigma}^{+}(X)$ and $M_{\sigma}^{+}(\nu X)$, because $\nu X$ is a strongly measure-compact Prohorov (or $T_{-}$) space [30]; every $\sigma$-additive Baire measure on $\nu X$ is of the form $\sum_{1}^{\infty} c_{n} \delta\left(x_{n}\right)$, where $x_{n} \in \nu X \forall n$ and $\sum_{1}^{\infty}\left|c_{n}\right|<\infty$. Then, by looking at "horizontal lines" in $\nu X$ and using [30, Th. 4.4] one can show that $H$ lives on $A \cup T \cup\{p\}=A \cup \beta T$, where $A$ is a subset of $D$ of the form $\left[0, \alpha_{0}\right] \times\left[0, \omega_{0}\right], \alpha_{0}<\omega_{1}$. Then $H \mid A$ is a weak*-compact subset of $M_{\tau}^{+}(D)$, and $H \mid \beta T$ is a weak*compact subset of $M^{+}(\beta T)$, so each can be pulled back to a weak*compact subset of $M_{\sigma}^{+}(E(X))$. The sum of these two pre-images contains the desired pre-image of $H$.

We do not know an example of a space with the WLP which is not $\beta_{1}$-stable.

\section{REFERENCES}

1. R. Alo and H. Shapiro, Normal Topological Spaces, Cambridge University Press, 1974.

2. A. Arhangel'skii, Bicompact sets and the topology of spaces, Trans. Moscow Math. Soc., 13 (1965), 1-62.

3. H. Bauer, Konservative Abbildungen lokal-kompakter Räume, Math. Ann., 138 (1959), $398-427$.

4. - Mesures avec un eimage donnée, Rev. Roum. de Math., 11 (1966), 747-752.

5. N. Dykes, Mappings and realcompact spaces, Pacific J. Math., 31 (1969), 347-358.

6. - Generalizations of realcompact spaces, Pacific J. Math., 33 (1970), 571-581.

7. J. Dugundji, Topology, Allyn and Bacon, Boston, 1966. 
8. B. A. Efimov, Extremally disconnected compact spaces and absolutes, Trans. Moscow Math. Soc., 23 (1970), 243-285.

9. J. Flachsmeyer, Normal and category measures on topological spaces, Proc. Third Prague Topological Symposium, 1971, 109-116.

10. Z. Frolik, A generalization of realcompact spaces, Czech. Math. J., 13 (1963), 127-138. 11. L. Gillman and M. Jerison, Rings of Continuous Functions, Springer-Verlag, New York, 1976.

12. A. Gleason, Projective topological spaces, Illinois J. Math., 2 (1958), 482-489.

13. M.Henriksen and J. Isbell, Some properties of compactifications, Duke Math. J., 25 (1958), 83-105.

14. H. Herrlich and J. van der Slot, Properties which are closely related to compactness, Ned. Akad. Wetensch. Proc. Ser., A70 (1967), 524-529.

15. J. Horne, Countable paracompactness and cb-spaces, Notices Amer. Math. Soc., 6 (1959), 629-630.

16. A. and C. Ionescu Tulcea, Topics in the Theory of Lifting, Springer-Verlag, New York, 1969.

17. H. Junnila, Metacompactness, paracompactness and interior-preserving open covers, Trans. Amer. Math. Soc., 249 (1979), 373-385.

18. A. Kato, Union of realcompact spaces and Lindelöf spaces, (preprint).

19. J. Knowle3, Measures on topological spaces, Proc. London Math. Soc., 17 (1967), 139-156.

20. G. Koumoullis, Some topological properties of spaces of measures, Notices Amer. Math. Soc., 25 (1978), A-239.

21. J. Kupka, A Short proof and generalization of a measure theoretic disjointization lemma, Proc. Amer. Math. Soc., 45 (1974), 70-72.

22. H. E. Lacey and D. Hebert, On supports of regular Borel measures, Pacific J. Math., 27 (1968), 101-118.

23. S. P. Lloyd, Two lifting theorems, Proc. Amer. Math. Soc., 42 (1974), 128-134.

24. J. Mack, On a class of countably paracompact spaces, Pros. Amer. Math. Soc., 16 (1965), 467-472.

25. J. Mack and D. Johnson, The Dedekind completion of $C(X)$, Pacific J. Math., 20 (1967), 231-243.

26. R. Maher, Sirong liftings and Borel liftings, Advances in Math., 13 (1974), 55-72.

27. E. Michael, The product of a normal space and a metric space need not be normal, Bull. Amer. Math. Soc., 69 (1963), 375-376.

28. W. Moran, The additivity of measures on completely regular spaces, J. London Math. Soc., 43 (1968), 633-639.

29. - Measures and mappings on topological spaces, Proc. London Math. Soc., 19 (1969), 493-508.

30. S. Mosiman and R. Wheeler, The strict topology in a completely regular setting: relations to topological measure theory, Canad. J. Math., 24 (1972), 873-890.

31. B. Ponomarev and L. Shapiro, Absolutes of topological spaces and their continuous maps, Russian Math. Surveys, 31 (1976), 138-154.

32. H. Rosenthal, On relatively disjoint families of measures, with some applications to Banach space theory, Studia Math., 37 (1970), 13-36.

33. H. Schaefer, Topological Vector Spaces, Macmillan, New York, 1966.

34. S. Scheinberg, Topologies which generate a complete measure algebra, Advances in Math., 7 (1971), 231-239.

35. D. Sentilles, Bounded continuous functions on a completely regular space, Trans. Amer. Math. Soc., 168 (1972), 311-336.

36. R. Sikorski, Boolean Algebras, Springer-Verlag, New York, 1969.

37. L. Steen and J. Seebach, Counterexamples in Topology, Second Edition, SpringerVerlag, New York, 1978.

38. D. Strauss, Extremally disconnected spaces, Proc. Amer. Math. Soc., 18 (1967), 
305-309.

39. A. Sultan, A general measure extension procedure, Proc. Amer. Math. Soc., 69 (1978), 37-45.

40. F. Tall, The density topology, Pacific J. Math., 62 (1976), 275-284.

41. F. Topsoe, Measure spaces connected by correspondences, Math. Scand., 30 (1972), $5-45$.

42. S. Ulam, Zur Masstheorie in der allgemeinen Mengenlehre, Fund. Math., 16 (1930), 140-150.

43. V. Varadarajan, Measures on topological spaces, Amer. Math. Soc. Transl., 48 (1965), 161-228.

44. J. von Neumann and M. Stone, The determination of representative elements in the residual classes of a Boolean algebra, Fund. Math., 25 (1935), 353-378.

45. N. Warren, Normality problems under perfect mappings, Notices Amer. Math. Soc., 20 (1973), A-455.

46. R. G. Woods, A Boolean algebra of regular closed subsets of $\beta X-X$, Trans. Amer. Math. Soc., 154 (1971), 23-36.

47. Co-absolutes of remainders of Stone-Čech compactifications, Pacific J. Math., 37 (1971), 545-560.

48. - Ideals of pseudocompact regular closed sets and absolutes of Hewitt realcompactifications, Gen. Top. Appl., 2 (1972), 315-331.

49. R. G. Woods and K. Hardy, On c-realcompact spaces and locally bounded normal functions, Pacific J. Math., 43 (1972), 647-656.

50. — A Tychonoff almost realcompactification, Proc. Amer. Math. Soc., 43 (1974), 200-208.

51. J. Worrell, The closed continuous images of metacompact topological spaces, Port, Math., 25 (1966), 175-179.

52. S. Graf, A selection theorem for Boolean correspondences, J. reine angew. Math., 295 (1977), 169-186.

53. G. Jameson, Ordered Linear Spaces, Springer-Verlag Lecture Notes, vol. 141, 1970. 54. J. Mack, Countable paracompactness and weak normality properties, Trans. Amer. Math. Soc., 148 (1970), 265-272.

55. R. G. Woods, Maps that characterize normality properties and pseudocompactness, J. London Math. Soc., (2) 7 (1973), 453-461.

Received July 14, 1978 and in revised form January 19, 1979. This research was partially supported by N. S. F. Grant \#MSC 77-02146.

\section{NORTHERN ILLINOIS UNIVERSITY}

DeKalb, IL 60115 


\section{PACIFIC JOURNAL OF MATHEMATICS}

\section{EDITORS}

Donald BABBITT (Managing Editor)

University of California

Los Angeles, CA 90024

HUGo RossI

University of Utah

Salt Lake City, UT 84112

C. C. MOORE and ANDREW OGG

University of California

Berkeley, CA 94720

\section{J. DuGUNDJI}

Department of Mathematics

University of Southern California

Los Angeles, CA 90007

R. FINN and J. Milgram

Stanford University

Stanford, CA 94305

ASSOCIATE EDITORS
E. F. BECKENBACH
B. H. NeumanN
F. WOLF
K. YoSHIDA

\section{SUPPORTING INSTITUTIONS}

\author{
UNIVERSITY OF BRITISH COLUMBIA \\ CALIFORNIA INSTITUTE OF TECHNOLOGY \\ UNIVERSITY OF CALIFORNIA \\ MONTANA STATE UNIVERSITY \\ UNIVERSITY OF NEVADA, RENO \\ NEW MEXICO STATE UNIVERSITY \\ OREGON STATE UNIVERSITY \\ UNIVERSITY OF OREGON
}

\author{
UNIVERSITY OF SOUTHERN CALIFORNIA \\ STANFORD UNIVERSITY \\ UNIVERSITY OF HAWAII \\ UNIVERSITY OF TOKYO \\ UNIVERSITY OF UTAH \\ WASHINGTON STATE UNIVERSITY \\ UNIVERSITY OF WASHINGTON
}

The Supporting Institutions listed above contribute to the cost of publication of this Journal, but they are not owners or publishers and have no responsibility for its content or policies.

Mathematical papers intended for publication in the Pacific Journal of Mathematics should be in typed form or offset-reproduced, (not dittoed), double spaced with large margins. Please do not use built up fractions in the text of the manuscript. However, you may use them in the displayed equations. Underline Greek letters in red, German in green, and script in blue. The first paragraph or two must be capable of being used separately as a synopsis of the entire paper. Please propose a heading for the odd numbered pages of less than 35 characters. Manuscripts, in triplicate, may be sent to any one of the editors. Please classify according to the scheme of Math. Reviews, Index to Vol. 39. Supply name and address of author to whom proofs should be sent. All other communications should be addressed to the managing editor, or Elaine Barth, University of California, Los Angeles, California, 90024.

50 reprints to each author are provided free for each article, only if page charges have been substantially paid. Additional copies may be obtained at cost in multiples of 50 .

The Pacific Journal of Mathematics is issued monthly as of January 1966. Regular subscription rate: $\$ 84.00$ a year (6 Vols., 12 issues). Special rate: $\$ 42.00$ a year to individual members of supporting institutions.

Subscriptions, orders for numbers issued in the last three calendar years, and changes of address should be sent to Pacific Journal of Mathematics, P.O. Box 969, Carmel Valley, CA 93924, U.S.A. Older back numbers obtainable from Kraus Periodicals Co., Route 100, Millwood, NY 10546.

PUBLISHED BY PACIFIC JOURNAL OF MATHEMATICS, A NON-PROFIT CORPORATION

Printed at Kokusai Bunken Insatsusha (International Academic Printing Co., Ltd.). 8-8, 3-chome, Takadanobaba, Shinjuku-ku, Tokyo 160, Japan.

Copyright (C) 1979 by Pacific Journal of Mathematics Manufactured and first issued in Japan 


\section{Pacific Journal of Mathematics}

\section{Vol. 82 , No. 2 \\ February, 1979}

Krishnaswami Alladi and Paul Erdős, On the asymptotic behavior of large prime

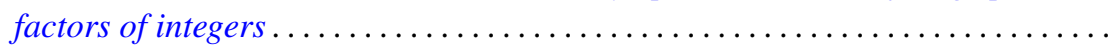

Alfred David Andrew, A remark on generalized Haar systems in $L_{p}$,

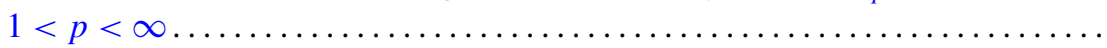

John M. Baker, A note on compact operators which attain their norm . . ........

Jonathan Borwein, Weak local supportability and applications to

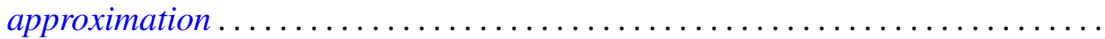

Tae Ho Choe and Young Soo Park, Wallman's type order compactification ........

Susanne Dierolf and Ulrich Schwanengel, Examples of locally compact

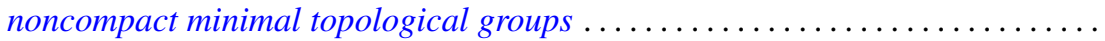

Michael Freedman, A converse to (Milnor-Kervaire theorem) $\times R$ etc. . . . . . . .

George Golightly, Graph-dense linear transformations ..................

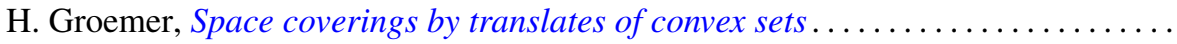

Rolf Wim Henrichs, Weak Frobenius reciprocity and compactness conditions in

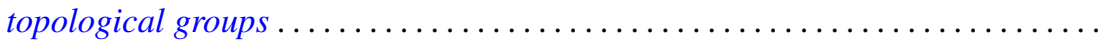

Horst Herrlich and George Edison Strecker, Semi-universal maps and universal

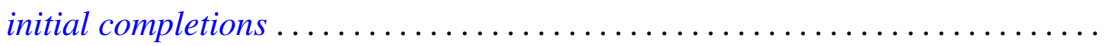

Sigmund Nyrop Hudson, On the topology and geometry of arcwise connected,

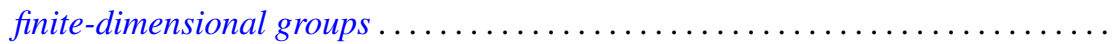

K. John and Václav E. Zizler, On extension of rotund norms. II .............

Russell Allan Johnson, Existence of a strong lifting commuting group of transformations. II.

Bjarni Jónsson and Ivan Rival, Lattice varieties covering the smallest nonmodular variety

Grigori Abramovich Kolesnik, On the order of Dirichlet L-functions .

Robert Allen Liebler and Jay Edward Yellen, In search of nonsolvable groups of

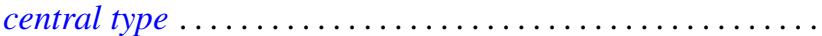

Wilfrido Martínez T. and Adalberto Garcia-Maynez Cervantes, Unicoherent plane Peano sets are $\sigma$-unicoherent ...

M. A. McKiernan, General Pexider equations. I. Existence of injective

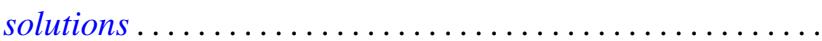

M. A. McKiernan, General Pexider equations. II. An application of the theory of webs.

Jan K. Pachl, Measures as functionals on uniformly continuous functions . .

Lee Albert Rubel, Convolution cut-down in some radical convolution algebras ...

Peter John Slater and William Yslas Vélez, Permutations of the positive integers

with restrictions on the sequence of differences. II . . .

Raymond Earl Smithson, A common fixed point theorem for nested spaces ....

Indulata Sukla, Generalization of a theorem of McFadden .... . . .

Jun-ichi Tanaka, A certain class of total variation measures of analytic measures.

Kalathoor Varadarajan, Modules with supplements .............. 